\title{
Percutaneous Mesh-container-plasty (PMCP) Versus Percutaneous Kyphoplasty (PKP) in the Treatment of Spinal Metastases: a Retrospective Cohort Study.
}

\section{Yimin Li}

Third Affiliated Hospital of Wenzhou Medical University

\section{Yunfan Qian}

Third affiliated Hospital of Wenzhou Medical University

\section{Guangjie Shen}

Third affiliated Hospital of Wenzhou Medical University

\section{Chengxuan Tang}

Third affiliated Hospital of Wenzhou Medical University

\section{Xiqiang Zhong}

Third affiliated Hospital of Wenzhou Medical University

Shaoqi HE ( $\sim$ heshaoqi@126.com )

Third affiliated Hospital of Wenzhou Medical University https://orcid.org/0000-0002-9368-2228

\section{Research Article}

Keywords: Percutaneous kyphoplasty, Percutaneous mesh-container-plasty, spinal metastases

Posted Date: February 1st, 2022

DOI: https://doi.org/10.21203/rs.3.rs-1278428/v1

License: (c) (i) This work is licensed under a Creative Commons Attribution 4.0 International License.

Read Full License 


\section{Abstract}

Background: To compare the clinical and radiological results of percutaneous mesh-container-plasty (PMCP) versus percutaneous kyphoplasty (PKP) in the treatment of spinal metastases.

Methods: Patients with confirmed diagnosis of spinal metastases and treated in our center between January 2011 and December 2019 were retrospectively included in the study. Ninety-four Patients were divided into 2 groups (PKP 50 and PMCP 44) according the surgical treatment they received. The epidemiological data, surgical outcomes, and clinical and radiological features were compared between the two groups. Cement leakage, height restoration, deformity correction, and cement distribution were calculated from the radiographs. The visual pain analog scale (VAS), Oswestry disability index (ODI), and Karnofsky performance scale (KPS) scores were calculated before surgery, and immediately and 2 years after surgery.

Results: The VAS, ODI, and KPS scores improved from 5 (4-6), 65.24 \pm 6.59 , and 40 (40-50) preoperatively to $2(1-2), 21.68 \pm 4.38$, and $70(70-80)$ immediately postoperatively in the PKP group ( $\mathrm{p}$ 0.05 ), and from $5(4-6), 66.45 \pm 6.04$, and $40(40-50)$ preoperatively to $1(1-2), 21.91 \pm 3.88$, and 70 (70$80)$ immediately postoperatively in the PMCP group $(p<0.05)$, respectively; there was no significant difference between the two groups. The mean cost in the PKP group was lower than that in the PMCP group ( $5563 \pm 439$ vs. $6569 \pm 344, p<0.05$ ). Cement distribution in the PMCP group was higher than that in the PKP group $(44.30 \% \pm 10.25 \%$ vs. $32.54 \% \pm 11.76 \%$, p < 0.05$)$. Cement leakage was observed to be lesser in the PMCP group $(7 / 44)$ than in the PKP group $(18 / 50, p<0.05)$. There were no statistically significant differences in the recovery of vertebral body height and improvement of segmental kyphosis between the PMCP and PKP groups in the treatment of spinal metastases.

Conclusions: Both PKP and PMCP have significant abilities in pain relief, functional recovery, height restoration, and segment kyphosis improvement. Despite its higher cost, PMCP may have a better ability to inhibit cement leakage and cement distribution than PKP in the treatment of spinal metastases.

\section{Background}

Bony metastases are a frequent problem in elderly patients with cancer. bony metastases involve the spine in approximately $50 \%$ of cases, and spinal metastases most often affect the vertebral bodies of the thoracic vertebrae $(70 \%)$, followed by the lumbar and cervical vertebrae ${ }^{[1,2]}$. The clinical presentation of metastatic spinal disease is predominantly pain, neurological deficit, progressive deformity, and general weakness. Pain is the most common symptom of spinal metastases, and more than $90 \%$ of patients complain of pain as an initial symptom ${ }^{[3]}$. Historically, spinal metastases were treated conservatively with chemotherapy, pain medications, physical therapy, and bracing. However, nonoperative treatment can result in continued deformity, progressive functional loss, and constant pain. Barzilai et al. ${ }^{[4]}$ proposed that treatment goals for patients with spinal metastases are palliative and include preservation 
or restoration of neurological function, improved pain control and health-related quality of life, and maintenance of spinal stability.

Recently, percutaneous kyphoplasty has emerged as a minimally invasive surgical treatment option for this patient population. Patients with spinal metastases without neurologic deficits who underwent percutaneous kyphoplasty demonstrated good clinical and radiological results. However, studies have shown some complications of percutaneous kyphoplasty, including cement leakage, loss of the restored height, and kyphotic alignment after balloon deflation before cement injection ${ }^{[5-7]}$. To avoid these complications, a mesh container was developed with the advantages of cement leakage, height restoration, and kyphotic angle reduction ${ }^{[8]}$.

Few reports have compared the curative effect of percutaneous kyphoplasty (PKP) and percutaneous mesh-container-plasty (PMCP) in the treatment of spinal metastases. We aimed to compare the clinical efficacy and safety of PMCP and PKP for the treatment of spinal metastases.

\section{Methods}

\section{Study design}

The study was approved by the ethics committee of the Third Affiliated Hospital of Wenzhou Medical University (YJ2020006).

The inclusion criteria were as follows: 1 ) elderly ( $\geq 55$ years), 2) diagnosed with unresectable malignant vertebral metastasis according to the criteria provided by Tokuhashi [9]; 3) Involvement of a single segment. 4) Underwent PKP or PMCP surgery and receive follow up visits up to 2 years after the surgery.

The exclusion criteria were: 1) age < 55years, 2) inability to tolerate surgery, 3) Involvement of multiple segments, 4) Less than 2 years of follow-up.

From January 2011 and December 2019, 94 patients who sustained spinal metastases without neurologic deficits were included in this study according to the inclusion and exclusion criteria. Vertebral biopsies were obtained during vertebroplasty for histological examination of each patient. Fifty patients underwent PKP and 44 patients underwent PMCP. The differences between PKP and PMCP were explained to all patients before surgery, and the surgical methods were selected according to patient preference.

Preoperatively, patients underwent electrocardiography (ECG), bleeding and clotting assessment, 3dimensional (3D) vertebral reconstruction scanned with computed tomography (CT), X-rays of the relevant spinal region in two planes, and magnetic resonance imaging. Patients aged $>65$ years additionally completed heart and lung function assessments. Standard clinical examination and evaluation, including medical history, physical examination of percussion pain, assessment of pain 
intensity (visual pain analog scale [VAS]), activity level (Oswestry disability index [ODI]), and Karnofsky performance scale (KPS), ${ }^{[10]}$ were evaluated.

\section{Surgical technique}

All operations were performed by the same senior chief physician under local anesthesia. The patients were positioned in the prone position on a radiolucent operating table, where four bolsters were placed with the abdomen freely suspended.

A 1-cm skin incision was made lateral to the desired entry point of the pedicle percutaneously. A trocar (Shandong Guanlong Medical Utensils Co., Ltd., Jinan City, Shandong Province, China) in a cannula was inserted into the pedicle at the treated vertebra through the pedicular approach as a working channel. After removing the trocar, a balloon was placed into the working channel and slowly inflated to create a low-pressure cavity for cement injection. The balloon continued to expand until the pressure reached 300 psi. The balloon was deflated and removed when the anteroposterior radiograph showed that the balloon size exceeded the midline of the vertebra; otherwise, a bilateral puncture was required.

In the PKP group, poly-(methyl methacrylate) (PMMA) cement was injected into the treated vertebra through the cannula under continuous fluoroscopic monitoring. PMMA insertion was considered complete when it reached the posterior third of the vertebral body or until the point at which cortical, epidural, and anterior venous cement leakage were considered possible.

In the PMCP group, a mesh container (Shandong Guanlong Medical Utensils Co., Ltd.) was advanced into the cavity. Thereafter, the PMMA cement was manually injected into the mesh container within the treated vertebra by applying a cement perfusion apparatus under fluoroscopic guidance. At a certain injection amount, the PMMA cement leaked outside of the mesh container from the meshes and entered the bone trabeculae (Figure 2).

A neurological examination was performed immediately after the operation. The patients were encouraged to walk while wearing a 3-point fixation brace after surgery. Radiographs and CT images were obtained to evaluate the reduction of the vertebra, improvement in segmental kyphosis, and distribution of the cement. Operation time, cost, hospital stay, and complications (cement leakage, cerebrospinal fluid leakage, and infection) were also noted. Back pain intensity was recorded using the VAS, functional outcome was evaluated using ODI, and long-term quality of life was assessed by KPS. All patients were postoperatively followed up clinically and radiologically immediately, and at 1, 3, and 6 months, and 1 and 2 years after surgery.

The Cobb angle and the anterior, middle, and posterior vertebral body height ratios (AVBHr, MVBHr, and $\mathrm{PVBHr}$, respectively) were measured using lateral radiographs as described in a previous study ${ }^{[11,12]}$. The cement distribution was calculated using CT images, and cement leakage was determined using X-ray CT images of the vertebra (Figure 1). 
Two independent blinded spine surgeons performed clinical evaluation of the patients, and three independent blinded spine surgeons assessed the radiographs.

\section{Statistical analysis}

Statistical analysis was performed using the Statistical Package for the Social Sciences version 19.0 software (IBM, Armonk, NY, USA). The numerical variables are presented as the mean \pm standard deviation or median (interquartile range). The Student's $t$-test or Wilcoxon signed-rank test was used to compare the measurements between the two groups. Repeated measures analysis of variance was used to compare the measurements of VAS, ODI, KPS, AVBHr, MVBHr, PVBHr, and the Cobb angle preoperatively, postoperatively, and 2 years postoperatively. The nominal variables (sex, cement leakage) are presented as numbers (percentages) and compared using the chi-square test. Statistical significance was set at a two-sided $p$-value of $<0.05$.

\section{Results}

Ninety-four Patients were divided into PKP group (50; 24 men, 26 women) and PMCP group (44; 21men, 23 women) according the surgical treatment they received. The clinical characteristics of the patients are summarized in Table 1. There were no statistical differences in the demographic data, including age and sex, between the two groups. We analyzed the segmental distribution and primary lesion of the involved vertebrae in the PKP and PMCP groups, and no statistically significant differences were found between the two groups (Table 1). All patients were followed up effectively, and no patients died or were lost to follow-up during the follow-up period. The mean cost in the PKP group was lower than that in the PMCP group ( $5563 \pm 439$ vs. $6569 \pm 344, p<0.05)$. There were no significant differences in operation time and hospital stay between the two groups. The details are presented in Table 2. 
Table 1

Basic characteristics and comparative analysis between PKP and PMCP for the treatment of the 94 patients with spinal metastases in this study $(x \pm s)$

\begin{tabular}{|c|c|c|c|c|}
\hline & $\operatorname{PKP}(n=50)$ & PMCP $(n=44)$ & $t\left(x^{2}\right)$ & $p$ \\
\hline Age(years) & $73.02 \pm 7.79$ & $74.68 \pm 7.88$ & $t=-1.026$ & 0.307 \\
\hline \multicolumn{5}{|l|}{ Sex } \\
\hline Male/female & $24 / 26$ & $21 / 23$ & $x^{2}=0.001$ & 0.979 \\
\hline \multicolumn{5}{|l|}{ Segments } \\
\hline T5-T9 & 10 & 7 & \multirow[t]{3}{*}{$x^{2}=0.348$} & \multirow[t]{3}{*}{0.840} \\
\hline T10-L2 & 28 & 27 & & \\
\hline L3-L5 & 12 & 10 & & \\
\hline \multicolumn{5}{|l|}{ Primary lesion } \\
\hline Respiratory system & 14 & 15 & \multirow[t]{4}{*}{$x^{2=} 2.191$} & \multirow[t]{4}{*}{0.534} \\
\hline Digestive system & 18 & 10 & & \\
\hline Urinary / Reproductive system & 12 & 14 & & \\
\hline Other sources & 6 & 5 & & \\
\hline
\end{tabular}

Table 2

Comparison of perioperative parameters between the PKP and PMCP groups for the treatment of the 94 patients with spinal metastases in this study $(x \pm s)$

\begin{tabular}{|lllll|}
\hline & PKP $(\mathbf{n}=50)$ & PMCP $(\mathbf{n}=\mathbf{4 4})$ & $t\left(\mathbf{x}^{2}\right)$ & $\boldsymbol{p}$ \\
\hline Operation time (min) & $31.00 \pm 4.40$ & $35.23 \pm 5.17$ & $\mathrm{t}=-4.283$ & $<0.001$ \\
\hline Hospital stay (days) & $11.46 \pm 9.52$ & $10.75 \pm 7.01$ & $\mathrm{t}=0.407$ & 0.685 \\
Cost (dollar) & $5563 \pm 439$ & $6569 \pm 344$ & $\mathrm{t}=-12.293$ & $<0.001$ \\
Cement leakage & $18 / 50$ & $7 / 44$ & $\mathbf{x}^{2}=4.839$ & 0.028 \\
\hline PKP: percutaneous kyphoplasty; PMCP: percutaneous mesh-container-plasty \\
\hline
\end{tabular}

\section{Clinical evaluation}


The VAS scores decreased from 5 (5-6) preoperatively to $2(1-2)$ postoperatively in the PKP group ( $\mathrm{p}<$ $0.05)$ and from $5(5-6)$ preoperatively to $2(1-2)$ postoperatively in the PMCP group $(p<0.05)$. The ODI scores decreased from $65.24 \pm 6.59$ preoperatively to $21.68 \pm 4.38$ postoperatively in the PKP group ( $p<$ $0.05)$ and from $66.45 \pm 6.04$ preoperatively to $21.91 \pm 3.88$ postoperatively in the PMCP group $(p<0.05)$. The KPS score improved from $40(40-50)$ preoperatively to $70(70-80)$ postoperatively in the PKP group $(p<0.05)$ and from $40(40-50)$ preoperatively to $70(70-80)$ postoperatively in the PMCP group $(p<$ 0.05). The follow-up results showed no significant changes in the VAS, ODI, and KPS scores 2 years after surgery. The differences in VAS, ODI, and KPS scores between the two groups were not statistically significant at postoperative and 2 years postoperatively. The details are presented in Table 3.

Table 3

Clinical comparisons between the PKP and PMCP groups for the treatment of the 94 patients with spinal metastases in this study $(\mathrm{x} \pm \mathrm{s}$ or median [interquartile range])

\begin{tabular}{|c|c|c|c|c|}
\hline & PKP $(n=50)$ & PMCP $(n=44)$ & $t(Z)$ & $p$ \\
\hline \multicolumn{5}{|l|}{ VAS } \\
\hline Preoperative & $5(4-6)$ & $5(4-6)$ & $Z=-0.852$ & 0.394 \\
\hline Postoperative & $2(1-2)^{\star}$ & $2(1-2) *$ & $Z=-0.585$ & 0.558 \\
\hline 2 years Postoperative & $1(1-2)^{*}$ & $1(1-2)$ * & $Z=-0.163$ & 0.871 \\
\hline 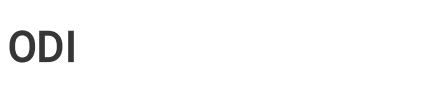 & & & & \\
\hline Preoperative & $65.24 \pm 6.59$ & $66.45 \pm 6.04$ & $\mathrm{t}=-0.927$ & 0.356 \\
\hline Postoperative & $21.68 \pm 4.38 *$ & $21.91 \pm 3.88 *$ & $t=-0.267$ & 0.790 \\
\hline 2 years Postoperative & $16.60 \pm 3.26^{*}$ & $16.45 \pm 3.11^{\star}$ & $t=0.221$ & 0.826 \\
\hline KPS & $40(40-50)$ & $40(40-50)$ & $Z=-0.648$ & 0.517 \\
\hline Postoperative & $70(70-80)^{\star}$ & $70(70-80)^{\star}$ & $Z=-0.042$ & 0.966 \\
\hline 2 years Postoperative & $80(70-80)^{*}$ & $80(70-80)^{\star}$ & $Z=-1.030$ & 0.303 \\
\hline \multicolumn{5}{|c|}{$\begin{array}{l}\text { * Repeated measures variance analysis was used for the statistical analysis. There were significant } \\
\text { differences }(p<0.05) \text { between the postoperative or } 2 \text { years postoperative and preoperative values of } \\
\text { these } 2 \text { groups. }\end{array}$} \\
\hline
\end{tabular}

PKP and PMCP showed significant pain relief and functional recovery in the treatment of spinal metastases at both postoperative and 2 years postoperatively, and there was no difference between the two groups. 


\section{Radiologic evaluation}

AVBHr, MVBHr, PVBHr, and Cobb angle scores improved in the PKP group from preoperative $(77.00 \% \pm$ $10.51 \%, 75.91 \% \pm 10.26 \%, 87.27 \% \pm 6.44 \%$, and $10.22 \% \pm 2.83 \%$, respectively) to postoperative $(81.48 \% \pm$ $9.50 \%, 81.59 \% \pm 9.20 \%, 89.04 \% \pm 5.79 \%$, and $7.14 \% \pm 2.20 \%)$ and from preoperative $(76.16 \% \pm 11.34 \%$, $73.49 \% \pm 11.57 \%, 86.92 \% \pm 7.06 \%$, and $10.20 \% \pm 2.21 \%)$ to postoperative $(83.56 \% \pm 8.93 \%, 82.18 \% \pm$ $8.37 \%, 89.20 \% \pm 5.96 \%$, and $6.43 \% \pm 1.52 \%)$ in the PMCP group ( $<0.05)$. Moreover, the long-term followup results showed that the AVBHr, MVBHr, PVBHr, and Cobb angle in the PKP and PMCP groups did not change significantly at 2 years postoperatively. There was no statistically significant difference in the improvement of $\mathrm{AVBHr}, \mathrm{MVBHr}$, and $\mathrm{PVBHr}$ and correction of the Cobb angle between the PMCP and PKP groups. The cement distribution in the PMCP group was significantly higher than that in the PKP group $(44.30 \% \pm 10.25 \%$ vs. $32.54 \% \pm 11.76 \%, p<0.05)$. All radiographic results are presented in Table 4 . There was no significant difference in the restoration of vertebral body height and improvement in segmental kyphosis between PKP and PMCP in the treatment of metastatic spinal tumors. The PMCP group had a significant advantage over the PKP group in terms of cement distribution. 
Table 4

Radiologic comparisons between the PKP and PMCP groups for treatment of the 94 patients with spinal metastases in this study $(\mathrm{x} \pm \mathrm{s})$
PKP $(n=50)$
$\operatorname{PMCP}(n=44)$
$t$
$p$

AVBHr (\%)

\begin{tabular}{lllll} 
Preoperative & $77.00 \pm 10.51$ & $76.16 \pm 11.34$ & 0.373 & 0.710 \\
\hline Postoperative & $81.48 \pm 9.50^{*}$ & $83.56 \pm 8.93^{*}$ & -1.091 & 0.278 \\
\hline 2 years Postoperative & $79.69 \pm 9.39 *$ & $82.49 \pm 9.03^{*}$ & -1.465 & 0.146
\end{tabular}

$\operatorname{MVBHr}(\%)$

Preoperative

$75.91 \pm 10.26$

$73.49 \pm 11.57$

1.071

0.287

Postoperative

$81.59 \pm 9.20$ *

$82.18 \pm 8.37 *$

$-0.327$

0.745

2 years Postoperative

$79.91 \pm 9.62 *$

$80.98 \pm 8.65 *$

$-0.560$

0.577

\section{PVBHr (\%)}

Preoperative

$87.27 \pm 6.44$

$86.92 \pm 7.06$

0.250

0.803

Postoperative

$89.04 \pm 5.79$ *

$89.20 \pm 5.96$ *

$-0.133$

0.894

2 years Postoperative

$88.12 \pm 5.75 *$

$88.56 \pm 6.01$ *

$-0.363$

0.717

\section{The Cobb angle $\left(^{\circ}\right)$}

Preoperative

$10.22 \pm 2.83$

$10.20 \pm 2.21$

0.029

0.977

Postoperative

$7.14 \pm 2.20$ *

$6.43 \pm 1.52$ *

1.791

0.077

2 years Postoperative

$7.88 \pm 2.45^{\star}$

$7.25 \pm 1.81$ *

1.404

0.164

Cement distribution (\%)

$32.54 \pm 11.76$

$44.30 \pm 10.25$

$-5.136$

$<0.001$

*Repeated measures variance analysis was used for the statistical analysis. There were significant differences $(p<0.05)$ between postoperative or 2 years postoperative and preoperative values of these 2 groups.

PKP: percutaneous kyphoplasty; PMCP: percutaneous mesh-container-plasty; AVBHr: anterior vertebral body height ratio; MVBHr: middle vertebral body height ratio; PVBHr: posterior vertebral body height ratio.

\section{Surgical complications}

A CT scan was performed immediately to assess cement leakage after surgery. Cement leakage was observed in $36 \%$ (18/50) of patients in the PKP group (four anterior to the vertebral body, four lateral to the vertebral, nine into the disk, and one into the canal without sequelae, Figure 2$)$ and in $16 \%(7 / 44)$ of patients in the PMCP group (two anterior to the vertebral body, two lateral to the vertebral and three into 
the disk without sequelae) $(\mathrm{p}<0.05)$ (Table 2). All cement leakages were asymptomatic, and no surgical intervention was required to remove the extravasated cement. Postoperative complications, such as neurological functional aggravation, hemorrhage, wound healing abnormalities, infection, and pulmonary embolism, were not observed during the 2-year follow-up period. These data suggest that PMCP is safer than PKP for controlling cement leakage in spinal metastases (Figure 3).

\section{Discussion}

Patients with spinal metastases are mostly in advanced stages of cancer and often present with complications such as pathological fractures of the vertebral body, spinal instability, spinal cord, and nerve root compression, which mainly present clinically as persistent and intractable pain and a gradual decrease in quality of life. In a retrospective study of patients with spinal metastases, Tomita et al. ${ }^{[13]}$ developed treatment goals and strategies based on the Tomita score, and palliative surgery was performed for patients with a score of 5 or higher. PKP has become an important treatment for vertebral metastases to relieve pain due to pathological fractures and metastatic tumor destruction of the vertebral body, as well as to prevent further tumor destruction of the diseased vertebral body, leading to continued collapse and loss of vertebral body height. Studies have shown that patients experienced significant pain relief of $80-98 \%$ within $48 \mathrm{~h}$ of percutaneous kyphoplasty, and its efficacy was evaluated mainly in terms of the degree of postoperative pain relief, maintenance of spinal stability, and improvement in quality of life ${ }^{[14,15]}$.

This study demonstrates that both PKP and PMCP techniques showed significant improvements in VAS, ODI, and quality of life in the treatment of spinal metastases, with no statistically significant differences. Furthermore, PMCP had better safety than PKP in terms of cement leakage and cement distribution ability.

Cement leakage is one of the most common complications of PKP, especially spinal cord and nerve root compression due to intravertebral cement leakage and lethal pulmonary embolism due to paravertebral vascular leakage. The leakage rate of spinal metastases tends to be higher than that of osteoporotic vertebral fractures alone, which may stem from tumor-induced destruction of the bone cortex of the vertebral body or tumor-rich vascularity and blood flow ${ }^{[16]}$. Sun et al. found that in the treatment of metastases, the leakage rate was $2.5 \%$ when the posterior wall of the vertebral body was intact, $5.1 \%$ when the non-posterior wall was defective, and $7.7 \%$ when the posterior wall of the vertebral body was locally fragmented and defective, and the leakage rate of bone cement with severe defects in the posterior wall was $13.3 \%{ }^{[17]}$. In order to reduce the risk of cement leakage,mesh containers were developed. Bone-filling mesh containers are dense mesh structures made of polyethylene terephthalate fibers with a tiny mesh. In the PMCP technique, a cavity is formed in the treated vertebral body by applying a bone expansion brace to cut the bone tissues. After withdrawal of the bone expansion brace, the mesh container is advanced into the cavity and PMMA cement is injected into the mesh container. During the cement injection process, the mesh container expands and reached the edge of the cavity. Continuous cement injection causes the mesh container to impart pressure on the surrounding bone 
tissues, and the height of the vertebral body is gradually restored. When the perfusion pressure reaches a certain degree, bone cement leaks outside of the mesh container from the meshes and enters the bone trabeculae, and, as a result, the bone trabeculae are strengthened and stabilized.

In this study, the leakage rate of bone cement in the PKP group was $36 \%(18 / 50)$ compared to $16 \%(7 / 44)$ in the PMCP group; therefore, for the treatment of metastatic spinal tumors, PMCP has a better ability to inhibit cement leakage than PKP.

Cement is a key factor in stabilizing the vertebral body, and the wider the distribution of cement, the better the surgical outcome, provided that the amount of cement ${ }^{[18]}$ remains constant ${ }^{[19-21]}$. Moreover, a reasonable cement distribution can reduce the risk of recompression after vertebroplasty ${ }^{[22]}$. It has been shown that adequate contact between the cement and the upper and lower endplates can better restore the strength of the vertebral body, maintain the height of the vertebral body, and reduce the risk of vertebral body recompression and long-term pain ${ }^{[23]}$. In this study, the cement distribution in the PMCP group was higher than that in the PKP group. Although we did not find significant differences in clinical outcomes between the two groups at the 2-year follow-up, the high distribution of bone cement in the vertebral body in the PMCP group may affect the maintenance of vertebral height, the improvement of segmental kyphosis, and the incidence of adjacent segmental fractures at long-term follow-up.

In addition, the recovery of vertebral body height and improvement of segmental kyphosis after treatment are important parameters for assessing the clinical efficacy of minimally invasive techniques. Previous studies found no correlation between the restoration of vertebral body height and improvement in clinical outcomes (e.g., pain reduction and functional recovery) ${ }^{[24,25]}$. In the present study, the recovery of vertebral body height and improvement in segmental kyphosis were higher in the PMCP group than in the PKP group. However, there were no statistically significant differences in clinical outcomes between the two groups. Considering that metastatic tumors of the spine are different from vertebral compression fractures, not all patients have significant vertebral compression changes and increased segmental kyphosis. Therefore, satisfactory results in the correction of vertebral body height and segmental kyphosis were achieved in both the PKP and PMCP groups, and there were no statistically significant differences.

Although PMCP has a greater advantage over PKP in the treatment of spinal metastases, caution is still required even with the mesh-container technique. The mesh container only delays the distribution of cement and reduces the risk of cement leakage, but does not eliminate the possibility of cement leakage. Because cement can diffuse outside the mesh bag through the mesh holes, premature leakage of cement out of the mesh bag and into the spinal canal or vasculature can occur when the cement is injected too thinly and too quickly, with catastrophic consequences. In this study, there were two cases with anterior leakage of the vertebral body, two cases with lateral leakage to the vertebral body and three cases with intervertebral leakage, but no symptoms such as neural compression were observed. Therefore, careful handling under $\mathrm{C}$-arm surveillance is still required to inject the cement slowly into the vertebral body during the drawing phase, to stop the injection as soon as the cement diffuses into the posterior third of 
the vertebral body, or if there is a potential trend of cement leakage from the cortex, epidural, and anterior veins.

The major shortcomings of this study are the small patient population and short follow-up duration. Prospective, randomized controlled studies enrolling more patients with long-term follow-up are needed in the future to evaluate the clinical and radiographic efficiency of PMCP more reliably and objectively.

\section{Conclusion}

Both PKP and PMCP have significant abilities in pain relief, functional recovery, height restoration, and segment kyphosis improvement. Despite its higher cost, PMCP may have a better ability to inhibit cement leakage and cement distribution than PKP in the treatment of spinal metastases.

\section{Abbreviations}

$\mathrm{PKP}=$ Percutaneous kyphoplasty, $\mathrm{PMCP}=$ Percutaneous mesh-container-plasty, $\mathrm{PMMA}=$ Poly-(methyl methacrylate), ODI = Oswestry disability index, VAS = visual analogue scale, KPS= Karnofsky performance scale, AVBHr=Anterior vertebral body height ratio, $\mathrm{MVBHr}=$ middle vertebral body height ratio, $\mathrm{PVBHr}=$ Posterior vertebral body height ratio

\section{Declarations}

\section{Ethics approval and consent to participate}

Ethics committee approval was received for this study from the Ethics Committee of Third Affiliated Hospital of Wenzhou Medical University (YJ2020006).

\section{Consent for publication}

\section{Availability of data and materials}

The datasets used and/or analyzed during the current study are available from the corresponding author on request.

\section{Competing interests}

The authors have no conflicts of interest to declare.

\section{Funding}

The authors declared that this study has received no financial support

\section{Authors' contributions}


Concept - S.H.; Design - Y.L., S.H.; Supervision - S.H.; Materials - X.Z.,Y.Q.; Data Collection and/or Processing - Y.Q., G.S.; Analysis and/or Interpretation - X.Z., G.S.; Literature Review - Y.L., S.H.; Writing Y.L.; Critical Review - Y.L., S.H.

\section{References}

1. Holman PJ, Suki D, McCutcheon I, Wolinsky JP, Rhines LD, Gokaslan ZL. Surgical management of metastatic disease of the lumbar spine: experience with 139 patients. J Neurosurg Spine. 2005;2(5):550-63.

2. Steinmetz MP, Mekhail A, Benzel EC. Management of metastatic tumors of the spine: strategies and operative indications. Neurosurgical focus. 2001;11(6):e2.

3. Helweg-Larsen S, Sørensen PS. Symptoms and signs in metastatic spinal cord compression: a study of progression from first symptom until diagnosis in 153 patients. Eur J Cancer. 1994;30a(3):396-8.

4. Barzilai O, Fisher CG, Bilsky MH. State of the Art Treatment of Spinal Metastatic Disease. Neurosurgery. 2018;82(6):757-69.

5. Kim YJ, Lee JW, Park KW, Yeom JS, Jeong HS, Park JM, et al. Pulmonary cement embolism after percutaneous vertebroplasty in osteoporotic vertebral compression fractures: incidence, characteristics, and risk factors. Radiology. 2009;251(1):250-9.

6. Nieuwenhuijse MJ, Van Erkel AR, Dijkstra PD. Cement leakage in percutaneous vertebroplasty for osteoporotic vertebral compression fractures: identification of risk factors. Spine J. 2011;11(9):83948.

7. Niu J, Zhou H, Meng Q, Shi J, Meng B, Yang H. Factors affecting recompression of augmented vertebrae after successful percutaneous balloon kyphoplasty: a retrospective analysis. Acta Radiol. 2015;56(11):1380-7.

8. Chen C, Li D, Wang Z, Li T, Liu X, Zhong J. Safety and Efficacy Studies of Vertebroplasty, Kyphoplasty, and Mesh-Container-Plasty for the Treatment of Vertebral Compression Fractures: Preliminary Report. PloS one. 2016;11(3):e0151492.

9. Tokuhashi Y, Matsuzaki H, Oda H, Oshima M, Ryu J. A revised scoring system for preoperative evaluation of metastatic spine tumor prognosis. Spine. 2005;30(19):2186-91.

10. Yıldız Çeltek N, Süren M, Demir O, Okan I. Karnofsky Performance Scale validity and reliability of Turkish palliative cancer patients. Turkish journal of medical sciences. 2019;49(3):894-8.

11. Thaler M, Lechner R, Nogler M, Gstöttner M, Bach C. Surgical procedure and initial radiographic results of a new augmentation technique for vertebral compression fractures. European spine journal : official publication of the European Spine Society, the European Spinal Deformity Society, and the European Section of the Cervical Spine Research Society. 2013;22(7):1608-16.

12. Xu C, Liu HX, Xu HZ. Analysis of related factors on the deformity correction of balloon kyphoplasty. AJNR American journal of neuroradiology. 2014;35(1):202-6. 
13. Tomita K, Kawahara N, Murakami H, Demura S. Total en bloc spondylectomy for spinal tumors: improvement of the technique and its associated basic background. Journal of orthopaedic science: official journal of the Japanese Orthopaedic Association. 2006;11(1):3-12.

14. Liu X, Yang Z, Xie L, Yuan Z, Ren M, Han L. Advances in the clinical research of the minimally invasive treatment for the posterior edge of vertebral-body defects by spinal metastases. Biomedical reports. 2015;3(5):621-5.

15. Wang Y, Liu H, Pi B, Yang H, Qian Z, Zhu X. Clinical evaluation of percutaneous kyphoplasty in the treatment of osteolytic and osteoblastic metastatic vertebral lesions. International journal of surgery (London, England). 2016;30:161-5.

16. Jensen ME, Kallmes DE. Percutaneous vertebroplasty in the treatment of malignant spine disease. Cancer journal (Sudbury, Mass). 2002;8(2):194-206.

17. Sun H, Yang Z, Xu Y, Liu X, Zhang Y, Chen Y, et al. Safety of percutaneous vertebroplasty for the treatment of metastatic spinal tumors in patients with posterior wall defects. European spine journal : official publication of the European Spine Society, the European Spinal Deformity Society, and the European Section of the Cervical Spine Research Society. 2015;24(8):1768-77.

18. Nieuwenhuijse MJ, Bollen L, van Erkel AR, Dijkstra PD. Optimal intravertebral cement volume in percutaneous vertebroplasty for painful osteoporotic vertebral compression fractures. Spine. 2012;37(20):1747-55.

19. He X, Li H, Meng Y, Huang Y, Hao DJ, Wu Q, et al. Percutaneous Kyphoplasty Evaluated by Cement Volume and Distribution: An Analysis of Clinical Data. Pain physician. 2016;19(7):495-506.

20. Liang D, Ye LQ, Jiang XB, Yang P, Zhou GQ, Yao ZS, et al. Biomechanical effects of cement distribution in the fractured area on osteoporotic vertebral compression fractures: a threedimensional finite element analysis. The Journal of surgical research. 2015;195(1):246-56.

21. Zhang L, Wang Q, Wang L, Shen J, Zhang Q, Sun C. Bone cement distribution in the vertebral body affects chances of recompression after percutaneous vertebroplasty treatment in elderly patients with osteoporotic vertebral compression fractures. Clinical interventions in aging. 2017;12:431-6.

22. Hou Y, Yao Q, Zhang G, Ding L, Huang H. Polymethylmethacrylate distribution is associated with recompression after vertebroplasty or kyphoplasty for osteoporotic vertebral compression fractures: A retrospective study. PloS one. 2018;13(6):e0198407.

23. Tan L, Wen B, Guo Z, Chen Z. The effect of bone cement distribution on the outcome of percutaneous Vertebroplasty: a case cohort study. BMC musculoskeletal disorders. 2020;21(1):541.

24. Feltes C, Fountas KN, Machinis T, Nikolakakos LG, Dimopoulos V, Davydov R, et al. Immediate and early postoperative pain relief after kyphoplasty without significant restoration of vertebral body height in acute osteoporotic vertebral fractures. Neurosurgical focus. 2005;18(3):e5.

25. McKiernan F, Faciszewski T, Jensen R. Does vertebral height restoration achieved at vertebroplasty matter? Journal of vascular and interventional radiology : JVIR. 2005;16(7):973-9.

\section{Figures}



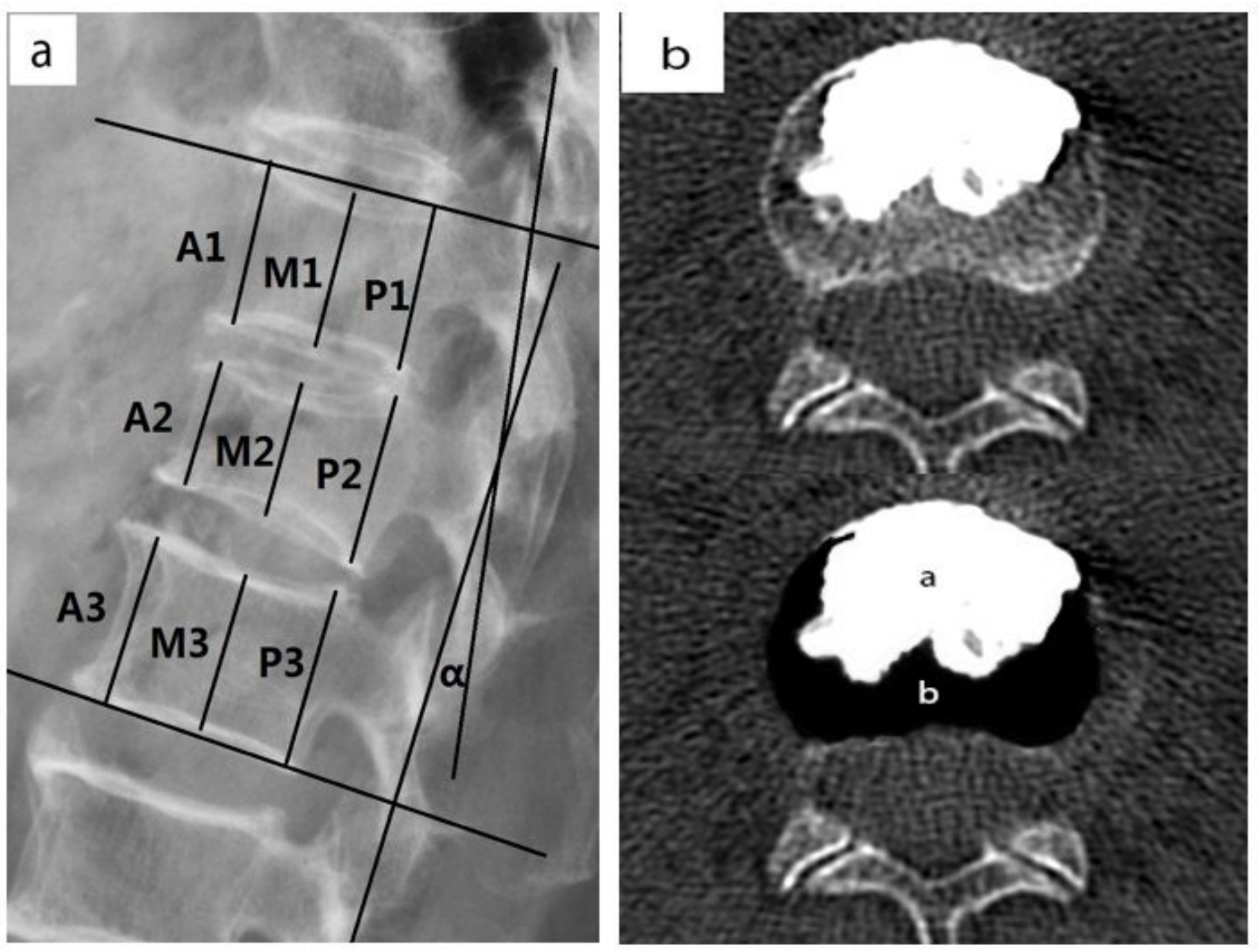

\section{Figure 1}

a-b. Methods of measurements on images. (a) Lateral radiograph showing evaluation of the Cobb angle, anterior vertebral body height ratio (AVBHr), middle vertebral body height ratio (MVBHr), and posterior vertebral body height ratio (PVBHr). The Cobb angle=a, AVBHr=2×A2 /(A1 +A3 ), MVBHr=2×M2 /(M1 +M3 ), and PVBHr=2×P2 /(P1 +P3 ). (b) Computed tomography (CT) plain image showing the evaluation of cement distribution. Cement distribution of a single CT section $=a /(a+b)$. Cement distribution was calculated as the mean of all CT sections of the treated vertebra. 

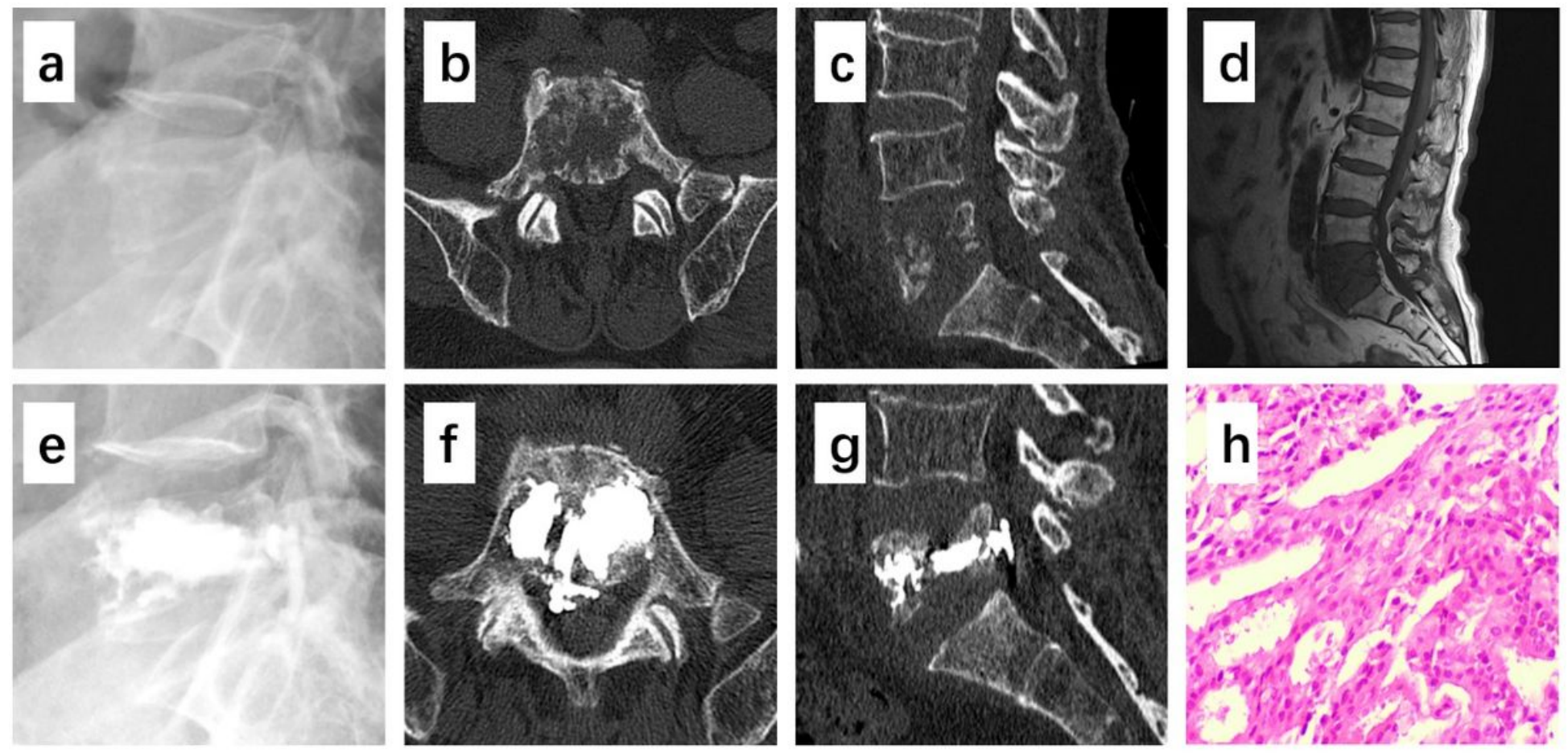

Figure 2

a-h. (a) Preoperative lateral radiograph showing a spinal metastases of L5. (b-c) Preoperative axial and sagittal CT scan showing a bone destruction in the L5 vertebral body. (d) Preoperative Lateral MRI showing a spinal metastases of L5. (e) Postoperative lateral radiograph showing cement leakage after undergoing PKP surgery of L5 spinal metastases. (f-g) Postoperative Axial and sagittal CT scan showing cement leakage after undergoing PKP surgery of L5 spinal metastases. (h)Postoperative pathological findings suggested metastatic adenocarcinoma of the L5 vertebral body, with a high probability of a prostate origin. 

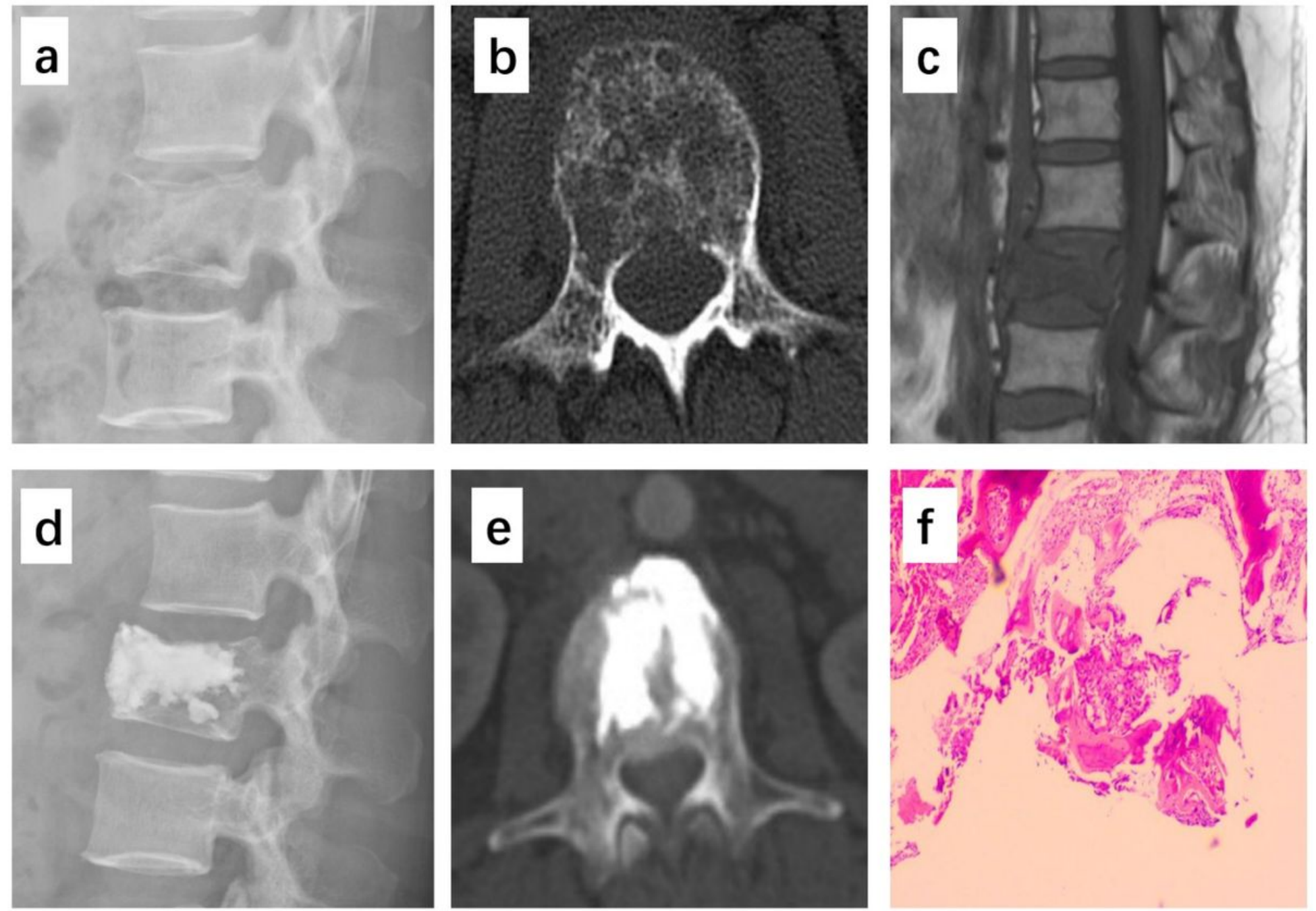

\section{Figure 3}

a-f. (a) Preoperative Lateral radiograph showing a spinal metastases of L3. (b) Preoperative computed tomography (CT) images showing bone destruction in the L3 vertebral body. (c) Preoperative magnetic resonance image (T1-weighted sequences) showing bone destruction in the L3 vertebral body. (d) Postoperative lateral radiograph after PMCP surgery. (e) Postoperative computed tomography (CT) images showing good distribution of cement in L3 vertebral body without leakage. (f)Postoperative pathology showed that the L3 vertebral trabeculae showed a nest of anomalous cells, which was consistent with a metastatic tumor. 